\title{
The 3-D Culture and In Vivo Growth of the Human Hepatocellular Carcinoma Cell Line HepG2 in a Self-Assembling Peptide Nanofiber Scaffold
}

\author{
Min Wu, Zehong Yang, Yanfei Liu, Bo Liu, and Xiaojun Zhao \\ Institute for Nanobiomedical Technology and Membrane Biology, West China Hospital, Sichuan University, Chengdu, 610041, China \\ Correspondence should be addressed to Xiaojun Zhao, xiaojunz@mit.edu
}

Received 24 March 2010; Revised 1 August 2010; Accepted 13 August 2010

Academic Editor: Jun Liu

Copyright (c) 2010 Min Wu et al. This is an open access article distributed under the Creative Commons Attribution License, which permits unrestricted use, distribution, and reproduction in any medium, provided the original work is properly cited.

\begin{abstract}
We report the use of the RADA16-I scaffold to mimic the ECM microenvironment and support tumor cell adherence and survival. Cellular morphology, proliferation, adhesion ability, and in vivo tumor formation were studied in the human hepatocellular carcinoma cell line HepG2 in the 3-D RADA16-I scaffold. No significant differences in HepG2 cell proliferation, adhesion, and albumin secretion were observed in the peptide scaffold compared to collagen I. Furthermore, the HepG2 cells precultured in the peptide scaffold showed a higher proliferation rate and formed significantly bigger tumors when compared to cells grown on a traditional 2D monolayer, suggesting that the 3-D RADA16-I scaffold can mimic the tumor microenvironment and promote a malignant phenotype in HepG2 cells. Our results indicate that the RADA16-I scaffold can serve as an ideal model for tumorigenesis, growth, local invasion, and metastasis.
\end{abstract}

\section{Introduction}

All tumor cells are embedded in a three-dimensional (3-D) microenvironment in the body. However, the majority of studies on the mechanisms of tumorigenesis have utilized cancer cell cultures on rigid surfaces. Results from many laboratories have shown dramatic changes in morphology and function depending on the presence of extracellular matrix. Tumorigenesis within a living organism results in distinct gene expression profiles compared to those of cancer cell lines $[1,2]$. Therefore, it is necessary to model the biological pathways and regulation of tumor cells in a true 3-D environment. In recent years, many synthetic polymer scaffolds, such as polylactide (PL), polyglycolide (PG), and poly (D, L-lactide-co-glycolide) (PLG), as well as natural matrix scaffolds, such as collagen, matrigel, and laminin, have been widely used to culture tumor cells [3]. However, there are many limitations to the actual application of these scaffolds in a cell culture laboratory; the synthetic polymer scaffolds are toxic and nonbiodegradable, while the animalderived biomaterials often contain residual growth factors, undefined constituents or nonquantified substances that make it difficult to conduct well-controlled studies with these materials.

An ideal 3-D culture system for tumor cells should be nontoxic, biodegradable, and well-defined. Previous studies have shown that the self-assembling peptide RADA16-I consists of natural amino acids that can undergo spontaneous assembly into nanofiber scaffolds of about $20 \mathrm{~nm}$ in diameter. This peptide can mimic the in vivo 3-D microenvironment and support the attachment of a variety of mammalian cells, including rat PC12 cells [4] and rat liver progenitor cells [5]. Moreover, this type of material has been extensively applied in regenerative medicine including brain reparation [6] and wound repair [7].

In the present study, we used self-assembling peptide nanofiber scaffolds to mimic the tumor microenvironment. To evaluate the effectiveness of the RADA16-I peptide scaffold in supporting attachment of HepG2 cells, maintaining cell proliferation and promoting a malignant phenotype, we assessed cell adhesion to the peptide scaffold, cell proliferation, and albumin secretion in a 3-D culture system. Furthermore, we compared the morphology and growth properties of HepG2 cells that had been precultured and 
encapsulated in a peptide scaffold prior to injection into SCID mice versus cells cultured in a traditional 2-D system.

\section{Materials and Methods}

2.1. Materials. The peptide RADA16-I ([COCH 3$]-$ RADARADARADARADA-[CONH$\left.{ }_{2}\right]$ ) solution (MW: 1,712) was commercially obtained from BD Biosciences, Bedford, under the trade name PuraMatrix. The concentration of the peptide was $1 \%(\mathrm{w} / \mathrm{v})$, and it had an approximately $4.0 \mathrm{pH}$. Calcein-AM was purchased from Molecular Probes. Rat tail collagen I $(1.57 \mathrm{mg} / \mathrm{mL})$ was purchased from BD Biosciences. Penicillin/streptomycin, Dulbecco's Modified Eagle Medium (DMEM), and fetal bovine serum (FBS) were obtained from Invitrogen. BSA was purchased from Sigma.

2.2. Self-Assembling Peptide Scaffold and AFM Assessment. Atomic force microscopy (AFM) was used to obtain the structure of the peptide. Five microliter aliquots of RADA16I peptide scaffold in Milli-Q water $(0.17 \mathrm{mg} / \mathrm{mL})$ were immediately deposited onto a freshly cleaved mica surface for $30 \mathrm{~s}$ and then rinsed with $100 \mu \mathrm{L}$ of Milli-Q water to remove the unattached peptide. The RADA16-I peptide solution on the mica surface was then air dried, and images were acquired immediately by scanning the mica surface by AFM (SPA400, SII Nanotechnology, Inc., Chiba, Japan) operating in tapping mode. Soft silicon cantilevers were chosen (force modulation etched silicon probe, Veeco Probes) with cantilever length of $219 \mu \mathrm{m}$, spring constant of $3 \mathrm{~N} / \mathrm{m}$, and curvature tip radius of $5 \mathrm{~nm}$. AFM scans were recorded at $512 \times 512$ pixels resolution and topographic images of the samples were produced, in which the brightness increases as a function of height. Images were collected at scales of $5 \times 5 \mu \mathrm{m}^{2}$ and $2 \times$ $2 \mu \mathrm{m}^{2}$, and measurements were repeated at least three times.

2.3. HepG2 Cells Cultured in 3-D Scaffold. The human Hepatocellular Carcinoma cell line HepG2 was maintained in the Dulbecco's Modified Eagle Medium (DMEM) with 10\% FBS and $1 \times$ penicillin/streptomycin. Before seeding the cells in the scaffold, cells were cultured on a monolayer to achieve $80 \%-85 \%$ confluency. Cells were then trypsinized, washed, collected by centrifugation, and resuspended in $20 \%$ sucrose. The cell suspension was mixed with an equal volume of $1 \%$ RADA16-I and loaded into the well, and then the medium was added slowly. The RADA16-I peptide self-assembled and formed a gel quickly, encapsulating tumor cells within the 3D nanofiber scaffold. The medium was changed three times in the next 30 minutes and then changed every two days. For analysis by light microscopy, the 3-D culture system was washed three times with PBS, fixed in $4 \%$ paraformaldehyde, dehydrated in a graded series of ethanol, and embedded in paraffin wax. $5 \mu \mathrm{m}$ thick serial sections were stained with hematoxylin and eosin ( $\mathrm{H} \& \mathrm{E})$. For analysis by fluorescence microscope, the 3-D culture system was washed with PBS once, fixed with $4 \%$ paraformaldehyde for 20 minutes, and then washed with PBS three times. Calcein-AM working solution $\left(2 \times 10^{-6} \mathrm{M}\right)$ was diluted in PBS for morphological assay of living cells. Images were obtained with an Olympus X 71 inverted microscope.

2.4. Proliferation and Metabolic Activity of HepG2. The viable cell number was determined by 3-(4, 5-dimethylthiazolyl-) 2, 5-diphenyltetrazolium bromide (MTT) assay for mammalian cell survival and proliferation [8]. HepG2 cells were seeded inside either the RADA16-I peptide scaffold $(n=4)$ or collagen I for 3, 7, and 12 days in 24-well culture plates. Culture medium was replaced with MTT $(0.5 \mathrm{mg} / \mathrm{mL}$ in PBS), and the cells were incubated for 4 hours. Samples from the culture plates were transferred to $1.5 \mathrm{~mL}$ tubes and centrifuged for 5 minutes at $8000 \mathrm{rpm}$. The purple formazan crystals were collected and dissolved in 10\% SDS with $0.01 \mathrm{M} \mathrm{HCl}$ at room temperature overnight. The solution from each sample was transferred into a microtiter plate. The absorbance at $490 \mathrm{~nm}$ was measured on a BioRad Immunoanalyzer. The albumin level was determined by a commercially available kit (HuaMei Bioengineering, Inc.). Briefly, HepG2 cells were cultured on either RADA16I peptide or collagen I for 3, 7, and 12 days, and the appropriate supernatants were transferred into $1.5 \mathrm{~mL}$ tubes $(n=4)$. Bromocresol green formed a colored complex with albumin that is detectable at $620 \mathrm{~nm}$. Known quantities of human albumin were used to establish the standard curve. Specific levels of albumin secretion were normalized to total protein levels (as determined by standard Bradford assay). Experiments were performed in triplicate.

2.5. Adhesion of HepG2 to Self-Assembling Peptide Scaffold. Adhesion assays of HepG2 to RADA16-I and collagen I were performed as previously described [9]. Ninety-six-well plates were coated with $50 \mu \mathrm{L}$ of RADA16-I, collagen I, and BSA ( $10 \mathrm{mg} / \mathrm{mL}, n=4$, respectively) by incubation overnight at $4^{\circ} \mathrm{C}$, and $50 \mu \mathrm{L}$ of PBS was added to initiate the self-assembly process of the peptide scaffold. The wells were washed twice with PBS, incubated with $10 \mathrm{mg} / \mathrm{mL}$ BSA for 30 minutes at room temperature to block uncoated surfaces, and rinsed twice with PBS before adding the cell suspension. For the adhesion assay, HepG2 cells were grown in a 2-D layer. The cell layer was rinsed with ice cold PBS before being scraped into PBS on ice. Cell suspensions $\left(5.0 \times 10^{3}\right.$ cells $)$ were added to each well previously coated with peptide scaffold, collagen I, or BSA, and cells were incubated in the medium supplemented with $1 \% \mathrm{FBS}$ at $37^{\circ} \mathrm{C}$ for 4 hours. The cell suspension was then removed from the well and rinsed twice with PBS. The viability was measured by MTT assay.

2.6. HepG2 Tumor Formation in Vivo. Experimental animal procedures were approved by the Laboratory Animal Welfare Committee on the Use of Animals in Research and Teaching. $1 \times 10^{7} \mathrm{HepG} 2$ cells precultured in the peptide scaffold or on 2-D petri dishes were collected and subcutaneously injected into the right groin of six-week-old female immunocompromised severe combined immunodeficiency (SCID) mice (State Key Lab of Biotherapy of Human Disease, Chengdu, China). At the end of the experiments, the SCID mice 


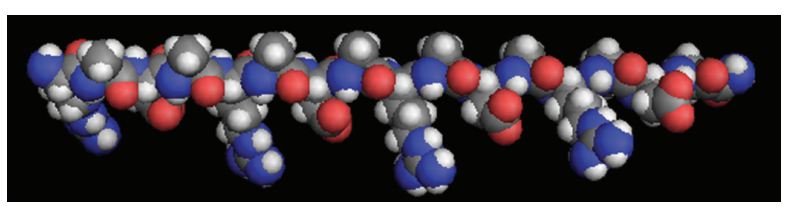

(a)

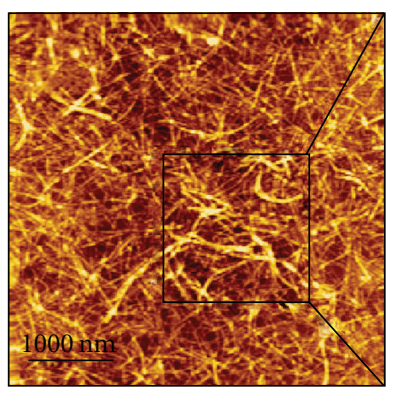

(b)

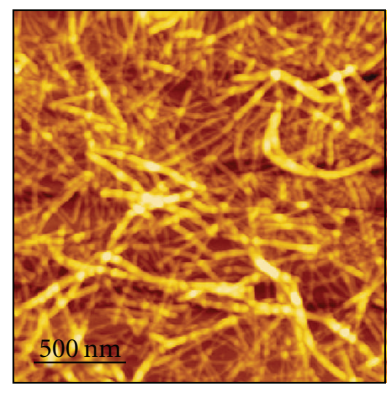

(c)

Figure 1: (a) Molecular model of RADA16-I. Carbon atoms are gray, oxygen atoms are red, nitrogen atoms are blue and hydrogen atoms are white. ((b)-(c)) AFM images of nanostructures formed from RADA16-I peptides. The bars represent $1000 \mathrm{~nm}$ and $500 \mathrm{~nm}$, respectively.

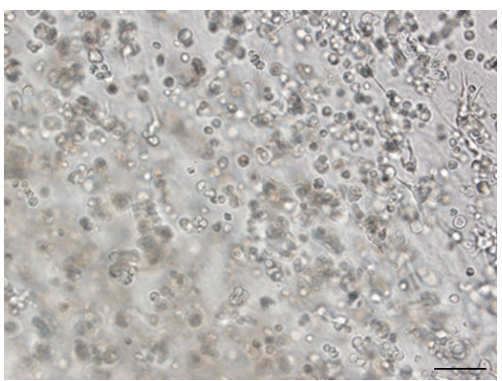

(a)

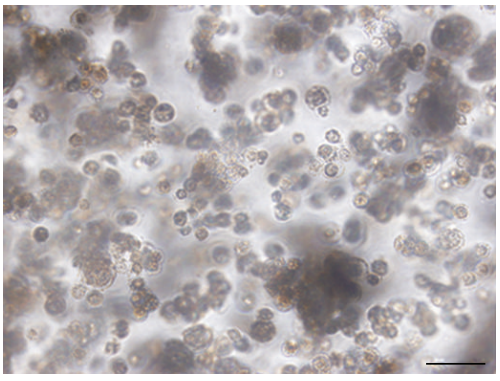

(c)

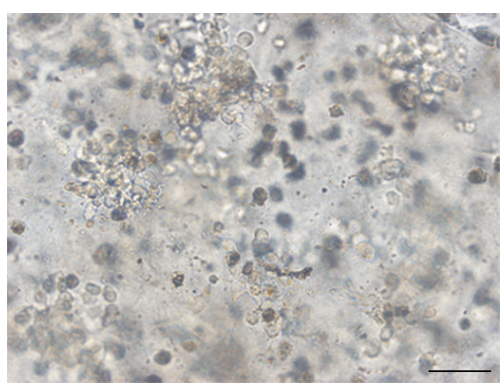

(b)

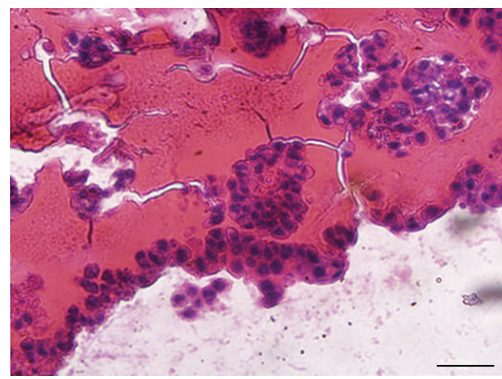

(d)

Figure 2: ((a)-(c)) Light microscopy images of HepG2 cells encapsulated in the RADA16-I scaffold for 3, 7, and 12 days. Bar = $200 \mu \mathrm{m}$. (d) Light microscopy evaluation of HepG2 cells in RADA16-I for 7 days, stained by hematoxylin and eosin $(\mathrm{H} \& \mathrm{E})$. Bars $=50 \mu \mathrm{m}$.

were sacrificed with ketamine $(3 \mathrm{~mL} / \mathrm{kg})$, the tumors were retrieved and weighed.

2.7. Statistical Methods. Student's T-test was used to test for statistical significance between experimental groups. The results are given as mean \pm standard deviation $(\mathrm{SD})$ with $P<0.05$ for significance.

\section{Results and Discussion}

3.1. AFM Assessment of Self-Assembling Peptide Nanofiber Scaffold. Self-assembling peptides are the synthetic nanobiomaterial derived from specific amino acid sequences in nature, and they have been widely used in tissue engineering and regenerative medicine [10]. These peptide nanofiber scaffolds are highly hydrated; they are composed of $99.5 \%$ 


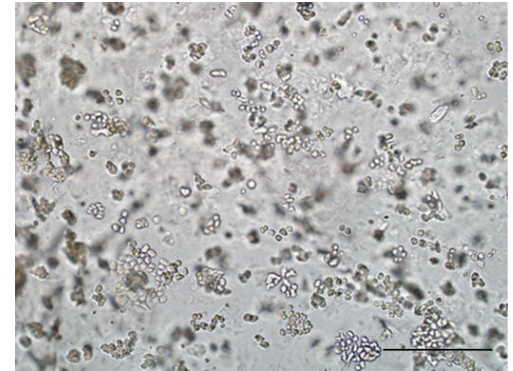

(a)

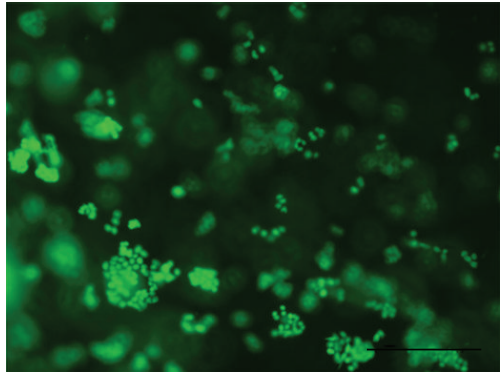

(b)

FIGURE 3: Planting of HepG2 cells in RADA16-I scaffold for 3 days. (a) Light microscopy images show that the cells maintained a spheroid form. (b) Living cells stained by calcein-AM for 3 days. Bars $=200 \mu \mathrm{m}$.

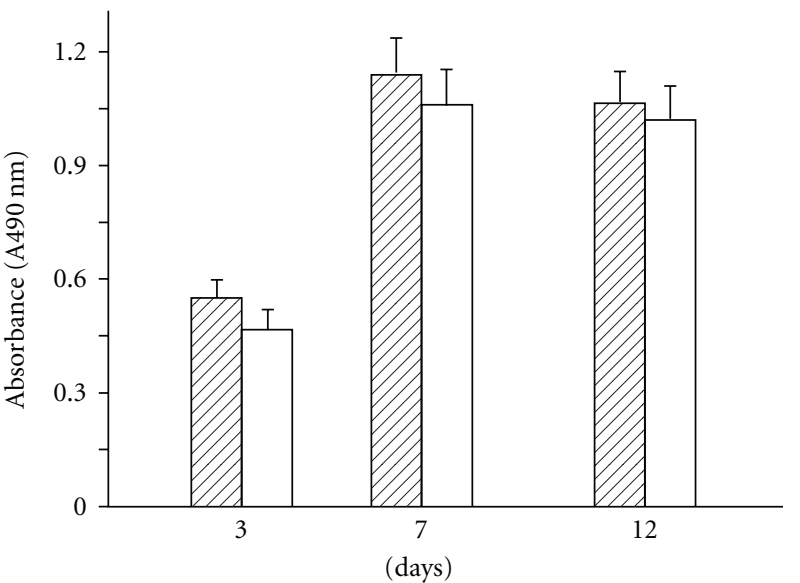

ZIJ RADA16-I Collagen I

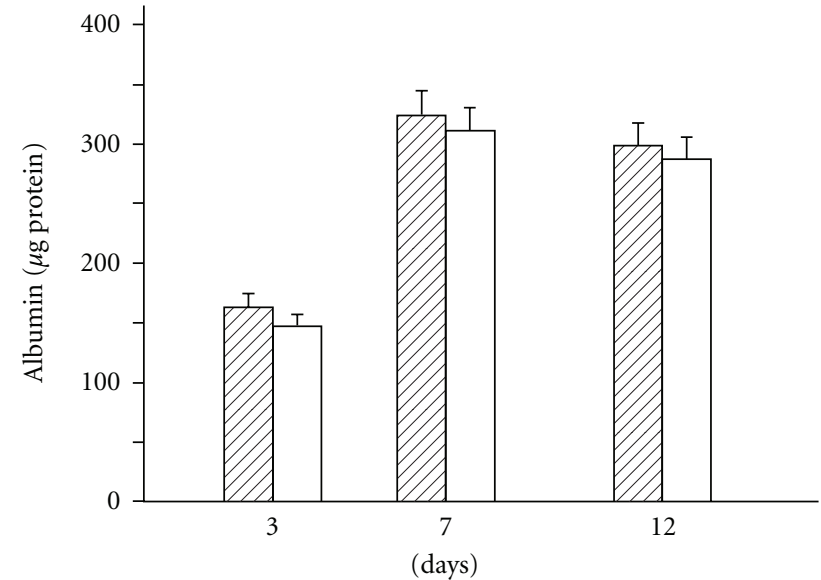

RADA16-I Collagen I

(a)

(b)

FIGURE 4: The proliferation and albumin secretion of HepG2 cells cultured in RADA16-I and collagen I for 3, 7, and 12 days. (a) MTT assay of HepG2 cells cultured on scaffolds. (b) Albumin secreted into supernatant. Albumin concentration was normalized to total amount of protein per well.

water. RADA16-I is an ionic self-complementary peptide that can form interwoven nanofiber networks ranging from a few hundred nanometers to a few microns $[4,11,12]$. A nanoscale molecular model is presented in Figure 1(a) as a building block for the RADA16-I peptide scaffold. AFM showed that the nanofiber scaffold consisted of nanofibers with lengths of $1619.83 \pm 90.16 \mathrm{~nm}$ and diameters of $32.37 \pm 1.72 \mathrm{~nm}$ (Figures $1(\mathrm{~b})$ and $1(\mathrm{c})$ ), which closely mimicked the porosity and gross structure of ECM, in which the cells could reside and migrate in the 3-D environment.

\subsection{The Morphology of the Human Hepatocellular Car-} cinoma Cell Line HepG2 in RADA16-I. The RADA16-I peptide scaffold was completely transparent, which was advantageous for collecting accurate images in this 3$\mathrm{D}$ culture system. To examine cell-scaffold constructions, we fabricated a 3-D culture system comprised of HepG2 cells within the RADA16-I peptide scaffold in 24-well cell culture plates (Figures 2(a)-2(d)). The HepG2 cells could intimately interact with the surrounding peptide scaffold. In this 3-D microenvironment, the morphologies of HepG2 cells changed sequentially with time, as recorded by phase contrast microscopy. They developed phenotypically from early single tumor cell to converged cell clusters on day 3 (Figure 2(a)) to multicellular spheroids on day 7 (Figures 2(c) and 2(d)). This morphological change suggests that the tumor cells could retain differentiated and active functional states within the RADA16-I peptide scaffold and that the interactions between HepG2 cells and the pericellular scaffolds are remodeled. Furthermore, the scaffold promoted the progression of differentiation during tumorigenesis in vitro (Figures 2(a)-2(d)). Additionally, most cells were alive in RADA16-I and formed multicellular colonies (Figures 3(a) and 3(b)). Similar conclusions have recently been reached by engineering tumors in other 3-D scaffolds [13]. 
3.3. Proliferation and Metabolic Activity of HepG2 Cells. The proliferation of HepG2 cells in the RADA16-I and collagen I scaffolds was analyzed by MTT assay for 3, 7, and 12 days (Figure 4(a)). Cell proliferation and distribution in the RADA16-I peptide scaffold were not significantly different from proliferation and distribution in the collagen I scaffold. Furthermore, HepG2 cells re-established cell-cell contacts and formed multicellular spheroids in the RADA16I peptide scaffold. However, we did not observe HepG2 cell aggregated in collagen I (data not shown). Recently, some studies have assessed the effectiveness of self-assembling peptide nanofibers as extracellular matrices (ECMs) in freshly isolated primary hepatocytes with similar results; selfassembling peptides maintained the differentiated functions, phenotypes, cell morphologies, and metabolic activities of hepatocytes in a 3-D configuration in vitro $[14,15]$.

The production of albumin is often used as an indicator of hepatocyte metabolic activity because albumin plays a critical role in the binding and transport of drugs, maintenance of colloid osmotic pressure, and scavenging of free radicals in vivo [16]. Figure 4(b) shows the concentration of albumin secreted by HepG2 cells cultured in the RADA16I or collagen I scaffold. The values have been normalized to account for any differences in cell number. It was evident that there was no significant difference in the supernatant derived from RADA16-I peptide scaffold compared to collagen $\mathrm{I}$, indicating that both 3-D microenvironments enhanced HepG2 cell function. It should be noted that the albumin secretion rate was greater than the previously reported values [17-19].

3.4. Adhesion of HepG2 to RADA16-I Scaffolds. Fibrin gels are commonly-used substrates for tumor cell adhesion studies [20]. Collagen is used to simulate the ECM because it is an important component of the ECM in vivo [21]. The adherence of HepG2 cells to RADA16-I, collagen I, and BSA scaffolds was analyzed to determine whether HepG2 cells have different affinities to the various substrates, which might indicate 3-D cell-cell and cell-microenvironment interactions. In our studies, the HepG2 cells showed similar binding affinities to collagen I and RADA16-I peptide scaffold, and both were significantly different when compared to the control BSA (Figure 5), suggesting that the RADA16-I peptide nanofiber scaffold contains biomimetic molecular structural characteristics also possessed by natural collagen I. The percent adhesion of HepG2 cells to collagen I and RADA16I was $28.55 \% \pm 6.06 \%$ and $27.39 \% \pm 5.47 \%$, respectively. It was evident that the RADA16-I peptide scaffold offered an ECM-like microenvironment for HepG2 cells to adhere to and proliferate, but the particular adhesive structures and mediators of adhesion remain to be identified in detail. These data show that the RADA16-I peptide nanofiber scaffold represents a new class of biologically-inspired material for cell adhesion and differentiation studies [22, 23].

3.5. Tumorigenesis of Precultured HepG2 Cells in 3-D or 2-D Systems. To determine the effects of the peptide nanofiber scaffold on the actual tumor formation and malignant

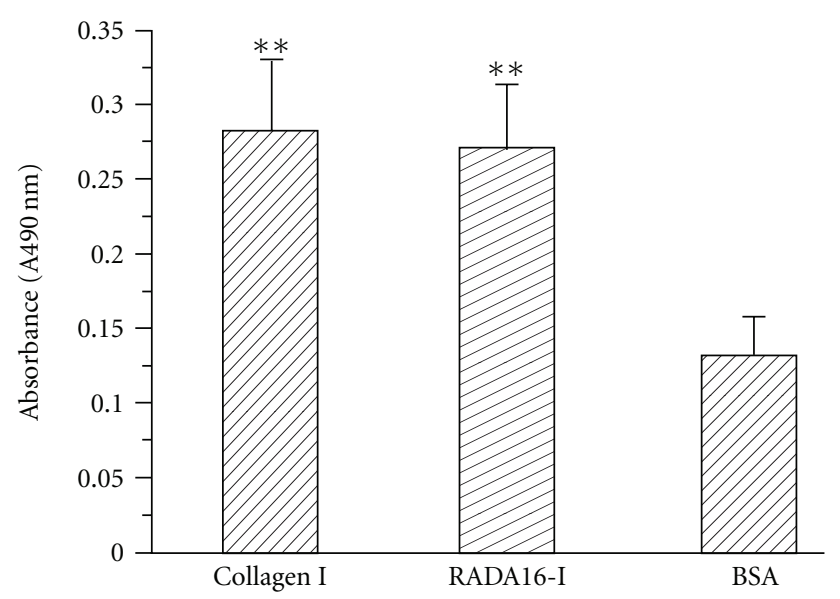

Figure 5: Adhesion of HepG2 cells to 3-D scaffold.

phenotype of HepG2 cells in vivo, we subcutaneously implanted equal numbers of HepG2 cells, precultured in either the RADA16-I scaffold or on 2-D petri dishes, into immunodeficient SCID mice. After sacrificing of the mice 28 days post-injection, the tumors were larger in the mice implanted with cells cultured in the $3-\mathrm{D}$ system in vitro (Figures 6(a) and 6(b)), suggesting that the culture of tumor cells in the 3-D peptide scaffold promoted more potent proliferation of HepG2 cells compared to the 2-D petri dish culture, which may contribute to the malignant phenotype of HepG2 cells. The RADA16-I scaffold could have also promoted the greater tumor weight and enhanced tumorigenicity of HepG2 Cells in vivo, compared to the 2D cell control (Figure 6(c)). It indicated that the 3-D cell culture peptide scaffold was biologically superior and that the in vivo microenvironment of tumorigenesis was more closely simulated compared to the traditional 2-D petri dish. This cell culture prototype is the preferred methodology to explore tumorigenesis in vitro.

\section{Conclusions}

Molecular self-assembly is ubiquitous in the natural world, and it is a powerful approach for fabricating novel supramolecular architectures. Self-assembling peptides present a variety of templates in nanotechnology and enable fabrication of many working prototypes for widespread use not only in cell culture but also in tissue repair, tissue engineering, and regenerative medicine. The RADA16-I peptide scaffold is a biocompatible scaffold for various cells, including differentiated stem cell progeny (i.e., progenitor cells, astrocytes, oligodendrocytes, and neurons) and freshly isolated primary cells (rat and porcine hepatocytes, osteoblasts, etc.) $[5,14,15,22,24,25]$. Our results suggest that HepG2 cells cultured in a 3-D peptide scaffold appear to simulate the conditions of HepG2 in vivo. The malignant phenotypes of HepG2 cells in peptide scaffold cultures had significantly higher rates of proliferation compared to the 2-D monolayer cultures. The RADA16-I peptide 


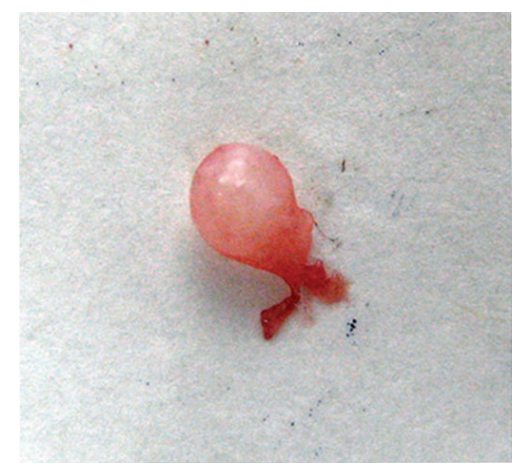

(a)

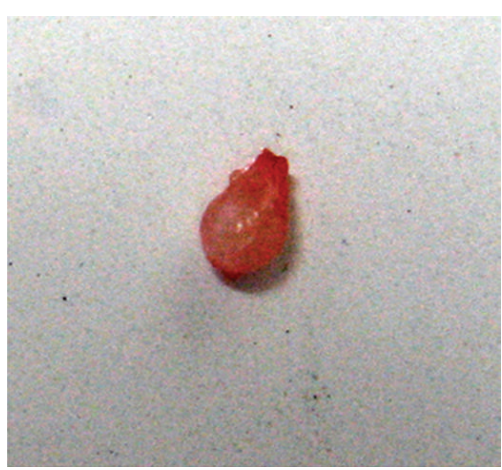

(b)

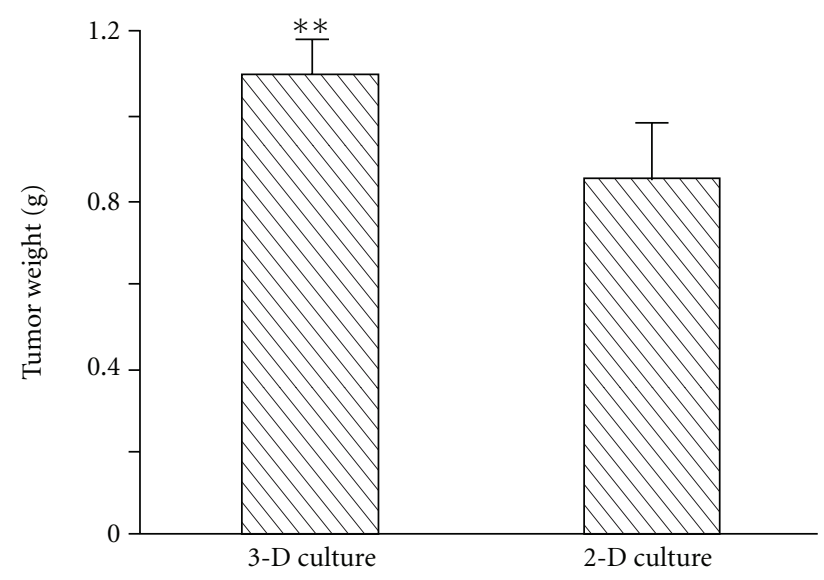

(c)

FIGURE 6: In vivo tumor proliferation of HepG2 cells precultured in 3-D scaffolds and 2-D petri dishes. ((a) and (b)) Images. (c) Weight.

scaffold allowed HepG2 cells to organize into cell-scaffold constructions and promoted the formation of cell clusters and multicellular spheroids. Moreover, the 3-D RADA16I peptide scaffold has obvious advantages over traditional 2-D culture systems. The 3-D scaffold also remodeled the extracellular matrix and cell-ECM interactions, which are very important in regulating homeostasis and tissue specificity. This novel nanofiber peptide scaffold may provide us with a powerful tool for both cancer research and the development of new diagnosis methods.

\section{Acknowledgment}

This research was financially and technically supported by the Chinese National "985 Project" of Education Ministry of Sichuan University, Chengdu, China.

\section{References}

[1] C. M. Perou, T. Sørile, M. B. Eisen, et al., "Molecular portraits of human breast tumours," Nature, vol. 406, no. 6797, pp. 747752, 2000.

[2] D. T. Ross and C. M. Perou, "A comparison of gene expression signatures from breast tumors and breast tissue derived cell lines," Disease Markers, vol. 17, no. 2, pp. 99-109, 2001.
[3] F. Xu and K. J. L. Burg, "Three-dimensional polymeric systems for cancer cell studies," Cytotechnology, vol. 54, no. 3, pp. 135143, 2007.

[4] T. C. Holmes, S. de Lacalle, X. Su, G. Liu, A. Rich, and S. Zhang, "Extensive neurite outgrowth and active synapse formation on self-assembling peptide scaffolds," Proceedings of the National Academy of Sciences of the United States of America, vol. 97, no. 12, pp. 6728-6733, 2000.

[5] C. E. Semino, J. R. Merok, G. G. Crane, G. Panagiotakos, and S. Zhang, "Functional differentiation of hepatocyte-like spheroid structures from putative liver progenitor cells in three-dimensional peptide scaffolds," Differentiation, vol. 71, no. 4-5, pp. 262-270, 2003.

[6] R. G. Ellis-Behnke, Y.-X. Liang, S.-W. You, et al., "Nano neuro knitting: peptide nanofiber scaffold for brain repair and axon regeneration with functional return of vision," Proceedings of the National Academy of Sciences of the United States of America, vol. 103, no. 13, pp. 5054-5059, 2006.

[7] H. Meng, L. Chen, Z. Ye, S. Wang, and X. Zhao, "The effect of a self-assembling peptide nanofiber scaffold (peptide) when used as a wound dressing for the treatment of deep second degree burns in rats," Journal of Biomedical Materials Research-Part B, vol. 89, no. 2, pp. 379-391, 2009.

[8] M. Bokhari, R. J. Carnachan, N. R. Cameron, and S. A. Przyborski, "Novel cell culture device enabling three-dimensional cell growth and improved cell function," Biochemical and Biophysical Research Communications, vol. 354, no. 4, pp. 1095-1100, 2007. 
[9] R. Kokenyesi, K. P. Murray, A. Benshushan, E. D. Huntley, and M.-S. Kao, "Invasion of interstitial matrix by a novel cell line from primary peritoneal carcinosarcoma, and by established ovarian carcinoma cell lines: role of cell-matrix adhesion molecules, proteinases, and E-cadherin expression," Gynecologic Oncology, vol. 89, no. 1, pp. 60-72, 2003.

[10] X. Zhao and S. Zhang, "Designer self-assembling peptide materials," Macromolecular Bioscience, vol. 7, no. 1, pp. 13-22, 2007.

[11] J. Guo, H. Su, Y. Zeng, et al., "Reknitting the injured spinal cord by self-assembling peptide nanofiber scaffold," Nanomedicine, vol. 3, no. 4, pp. 311-321, 2007.

[12] S. Zhanga, Y. Lin, M. Altman, et al., "Biological surface engineering: a simple system for cell pattern formation," Biomaterials, vol. 20, no. 13, pp. 1213-1220, 1999.

[13] C. Fischbach, R. Chen, T. Matsumoto, et al., "Engineering tumors with 3D scaffolds," Nature Methods, vol. 4, no. 10, pp. 855-860, 2007.

[14] N. Navarro-Alvarez, A. Soto-Gutierrez, J. D. Rivas-Carrillo, et al., "Self-assembling peptide nanofiber as a novel culture system for isolated porcine hepatocytes," Cell Transplantation, vol. 15, no. 10, pp. 921-927, 2006.

[15] S. Wang, D. Nagrath, P. C. Chen, F. Berthiaume, and M. L. Yarmush, "Three-dimensional primary hepatocyte culture in synthetic self-assembling peptide hydrogel," Tissue Engineering Part A, vol. 14, no. 2, pp. 227-236, 2008.

[16] C. S. Ranucci, A. Kumar, S. P. Batra, and P. V. Moghe, "Control of hepatocyte function on collagen foams: sizing matrix pores toward selective induction of 2-D and 3-D cellular morphogenesis," Biomaterials, vol. 21, no. 8, pp. 783793, 2000.

[17] C. S. Ranucci and P. V. Moghe, "Polymer substrate topography actively regulates the multicellular organization and liverspecific functions of cultured hepatocytes," Tissue Engineering, vol. 5, no. 5, pp. 407-420, 1999.

[18] R. Sudo, T. Mitaka, M. Ikeda, and K. Tanishita, "Reconstruction of 3D stacked-up structures by rat small hepatocytes on microporous membranes," The FASEB Journal, vol. 19, no. 12, pp. 1695-1697, 2005.

[19] H. Baharvand, S. M. Hashemi, S. K. Ashtiani, and A. Farrokhi, "Differentiation of human embryonic stem cells into hepatocytes in 2D and 3D culture systems in vitro," International Journal of Developmental Biology, vol. 50, no. 7, pp. 645-652, 2006.

[20] S. Even-Ram and K. M. Yamada, "Cell migration in 3D matrix," Current Opinion in Cell Biology, vol. 17, no. 5, pp. 524-532, 2005.

[21] B. A. Roeder, K. Kokini, J. E. Sturgis, J. P. Robinson, and S. L. Voytik-Harbin, "Tensile mechanical properties of threedimensional type I collagen extracellular matrices with varied microstructure," Journal of Biomechanical Engineering, vol. 124, no. 2, pp. 214-222, 2002.

[22] F. Gelain, A. Lomander, A. L. Vescovi, and S. Zhang, "Systematic studies of a self-assembling peptide nanofiber scaffold with other scaffolds," Journal of Nanoscience and Nanotechnology, vol. 7, no. 2, pp. 424-434, 2007.

[23] M. S. Kim, J. H. Yeon, and J.-K. Park, "A microfluidic platform for 3-dimensional cell culture and cell-based assays," Biomedical Microdevices, vol. 9, no. 1, pp. 25-34, 2007.

[24] A. Horii, X. Wang, F. Gelain, and S. Zhang, "Biological designer self-assembling peptide nanofiber scaffolds significantly enhance osteoblast proliferation, differentiation and 3D migration," PLoS ONE, vol. 2, no. 2, article e190, 2007.
[25] S. Wang, D. Nagrath, P. C. Chen, F. Berthiaume, and M. L. Yarmush, "Three-dimensional primary hepatocyte culture in synthetic self-assembling peptide hydrogel," Tissue Engineering Part A, vol. 14, no. 2, pp. 227-236, 2008. 

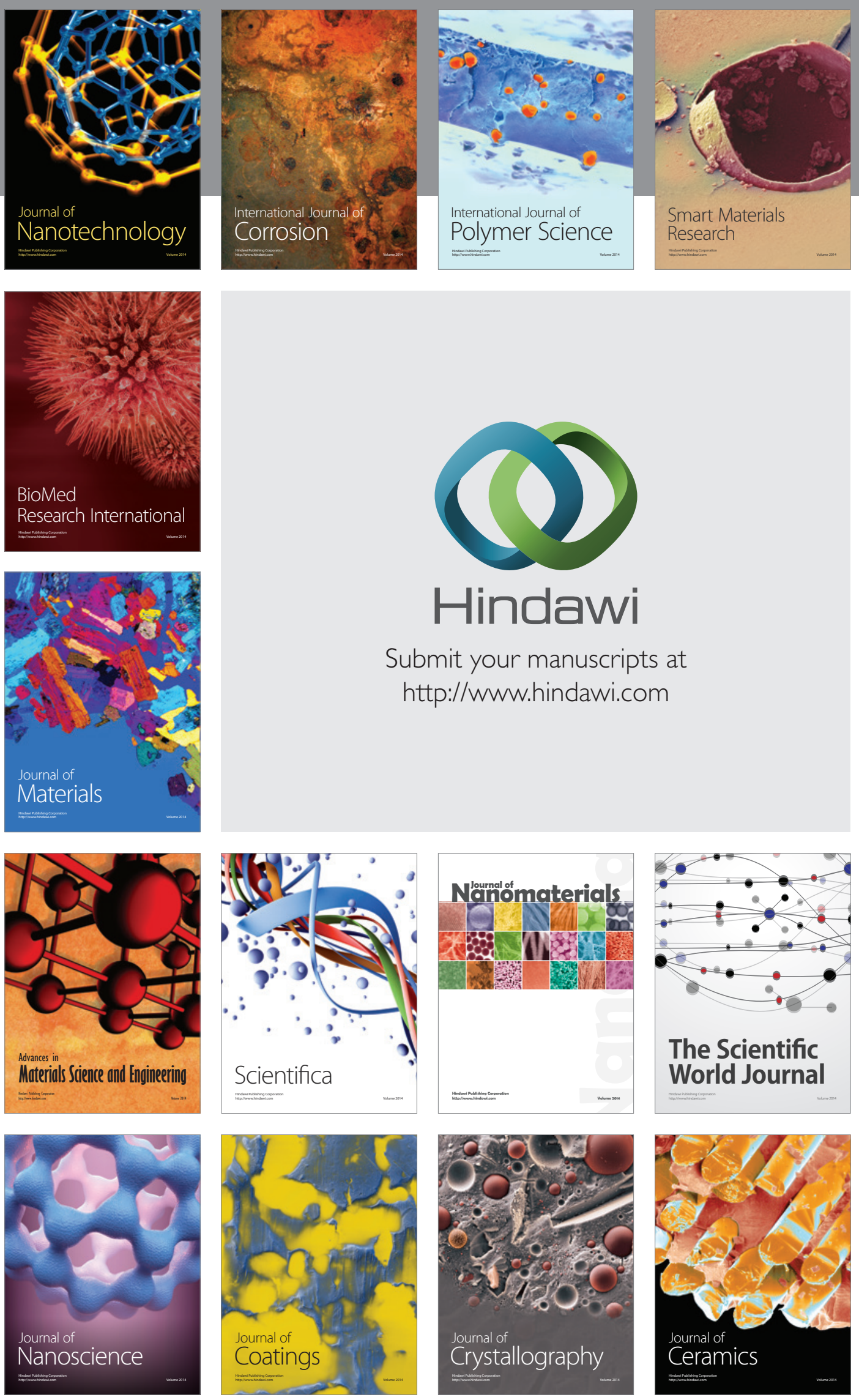

The Scientific World Journal

Submit your manuscripts at

http://www.hindawi.com

\section{World Journal}

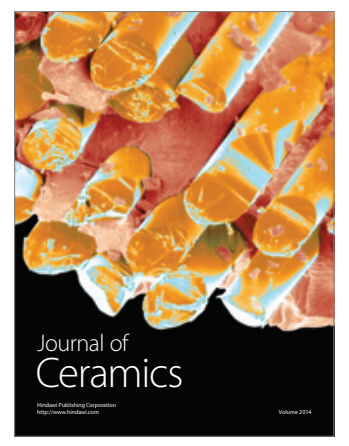

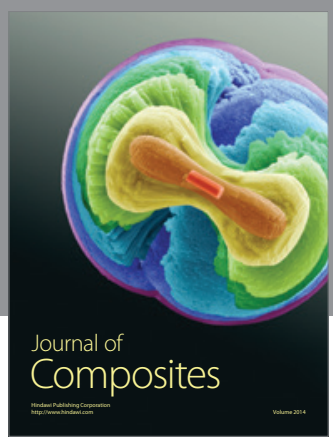
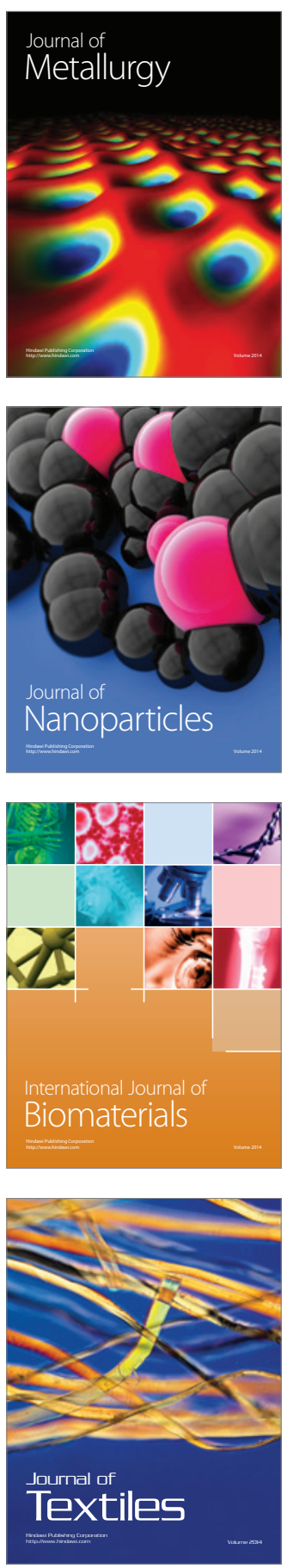